\title{
Aprimoramento curricular na perspectiva do desenvolvimento docente - desafios e possibilidades
}

\author{
Curricular enhancement from the perspective of faculty development - \\ challenges and possibilities
}

\section{Perfeccionamiento curricular desde la perspectiva del desarrollo docente - retos y oportunidades}

\author{
Anna Tereza M. S. de Moura," Albanita V. de Oliveira
}

\begin{abstract}
Resumo
A reflexão sobre o que e como ensinar nas escolas médicas se intensificou nas últimas décadas, com necessidade de adequação da matriz curricular ao perfil do profissional que a sociedade deseja e de que necessita na atualidade. Várias são as possibilidades para estruturar um currículo que seja contemporâneo e sintonizado com as demandas de saúde da população, o que torna o processo ainda mais desafiante. Para uma escola tradicional, com organização fragmentada de disciplinas e dificuldade de integração vertical e horizontal, essa tarefa pode ser ainda mais complexa. Promover a inserção dos alunos em diferentes cenários de prática, utilizando metodologias ativas de ensino e adotar ferramentas de avaliação formativas e sistematizadas são outros avanços apontados nas normativas atuais relacionadas ao ensino médico. Existe uma aparente distância entre os docentes e o ensino na graduação. A ausência de dispositivos claros para a meritocracia acadêmica parece ser um limitador da mobilização e da participação destes profissionais nas transformações necessárias. A literatura aponta para a possibilidade de alcançar reformas curriculares a partir de programas de desenvolvimento docente. A Faculdade de Ciências Médicas da Universidade do Estado do Rio de Janeiro se caracteriza como uma escola de perfil tradicional ainda buscando sua adequação às Diretrizes Curriculares em vigor. Para resgatar o processo de aprimoramento curricular, a direção organizou atividades para mobilizar seu corpo docente, avaliando que sem a legitimação deste grupo qualquer iniciativa de mudança não seria exitosa. Diferentes frentes de trabalho foram organizadas e a presença de docentes já sensibilizados, experiências exitosas prévias e apoio da gestão foram identificados como fatores positivos que vêm facilitando o processo. A resposta às iniciativas tem sido encorajadora, com a percepção de um movimento crescente de valorização das práticas na graduação e integração institucional.
\end{abstract}

Descritores: Currículo; Docentes; Educação de graduação em medicina.

\begin{abstract}
Reflection on what to teach and how to do it in medical schools has intensified in recent decades, with necessity to develop curriculum adapted to form professional who will attempt what society wants and needs nowadays. There are several possibilities to structure a curriculum that is contemporary and appropriated with health demands, which makes the process even more challenging. For a traditional school with disciplines organized in a fragmented way, and no vertical or horizontal integration, this task can be even more complex. Promoting the inclusion of students in different practice scenarios, using active methods of teaching and formative assessment tools are other advances pointed out in current policies related to medical education. There is an apparent distance form faculties and undergraduate teaching. The lack of clear landmarks for academic meritocracy seems to be limiting mobilization and participation of these professionals in the necessary transformations. The literature points out to the possibility of achieving curricular reform from faculty development programs. The Faculty of Medical Sciences from State University of Rio de Janeiro is characterized as a traditional school profile, still seeking its suitability to actual Curriculum Guidelines. To redeem the process of curriculum improvement, institutional manager developed activities to mobilize faculties, assessing that without legitimacy of this group any initiative would not be successful. Different task forces were organized and the presence of teachers who are already
\end{abstract}


sensitized, previous successful experiences and management support were identified as positive factors that facilitate the entire process. The response to the initiative has been encouraging, with perception of a growing movement of valuation practices for under graduation and institutional integration.

Keywords: Curriculum; Faculty; Education, Medical, Undergraduate.

\section{Resumen}

La reflexión sobre qué es y cómo enseñar en las escuelas de medicina se intensificó en las últimas décadas, con la necesidad de adecuar el plan de estudios al perfil del profesional que la sociedad desea y del que necesita en la actualidad. Existen varias formas para estructurar un plan de estudios que sea contemporáneo, amplio y en sintonía con las demandas de salud de la población, lo que vuelve el proceso aún más desafiante. Para una escuela tradicional, con organización fragmentada de disciplinas y dificultad de integración vertical y horizontal, esa tarea puede ser todavía más compleja. Promover la inclusión de los estudiantes en diferentes escenarios de la práctica, utilizando metodologías de enseñanza activas y adoptar herramientas de evaluación formativas y sistemáticas son otros avances necesarios. Existe una aparente brecha entre los docentes y la enseñanza en la graduación. La falta de dispositivos claros para la meritocracia académica parecer estar limitando la movilización y la participación de estos profesionales en las transformaciones necesarias para la adecuación del plan de estudios a los nuevos dispositivos relacionados con la enseñanza de medicina. La Facultad de Ciencias Medicas (FCM) de la Universidad del Estado de Rio de Janeiro (UERJ) reconoce la necesidad de movilizar e introducir su cuerpo docente en el proceso de perfeccionamiento curricular, evaluando que sin la legitimización de este grupo cualquier iniciativa de cambio no tendrá éxito. La organización de diferentes frentes de trabajo, la presencia de docentes ya sensibilizados, las experiencias exitosas previas y el apoyo de la gestión son factores positivos que vienen facilitando el proceso. La respuesta a las iniciativas ha sido alentadora, con la percepción de un movimiento creciente de valorización para prácticas de la graduación e integración institucional.

Palabras clave: Currículo; Docentes; Educación de graduación en medicina.

\section{Introdução}

A Faculdade de Ciências Médicas (FCM) da Universidade do Estado do Rio de Janeiro (UERJ) iniciou suas atividades no ano de 1936 e seus fundadores eram oriundos de distintas instituições à época, com objetivos afinados com o desenvolvimento da educação de nível superior no país. Em sintonia com os preceitos vigentes naqueles tempos, a FCM também atuava a partir do modelo proposto por Flexner, tendo seu ensino baseado na atenção desenvolvida principalmente no âmbito hospitalar.' É possível identificar movimentos de mudança ao longo dos mais de sessenta anos de sua trajetória que, porém, permanece sendo considerada uma faculdade de ensino tradicional e que pouco incorporou dos dispositivos apresentados pelas Diretrizes Curriculares do Ministério de Educação e Cultura (MEC) de 2001. ${ }^{2}$ Os caminhos percorridos apontam para inúmeras iniciativas de transformação, privilegiando sempre a formação de um egresso que se diferencia por uma visão ampliada sobre os determinantes de saúde e por forte base clínica. Todos estes movimentos tiveram participação dos corpos docente e discente e suas lideranças, contribuindo para a avaliação positiva que a FCM possui até os dias de hoje. ${ }^{3}$

Mas os tempos atuais são outros e demandam ajustes significativos no campo da educação médica. As diretrizes do início da década deram lugar às normativas estruturadas com base de lei, com ampliação do controle e avaliações externas sobre a organização dos cursos. ${ }^{4}$ Como sempre, o momento atual pode ser visto sob perspectivas políticas diversas, positivas ou não. Contudo, parece inegável que as reflexões recentes trouxeram à tona a necessidade de importantes transformações e a FCM não poderia ficar à margem de todo este movimento.

Para a formação de profissionais capazes de atuar em situações complexas e em diferentes cenários de saúde é preciso privilegiar atividades que envolvam a participação do aluno na construção do seu conhecimento a partir do desenvolvimento de práticas reflexivas. ${ }^{5}$ Nas escolas tradicionais, estas iniciativas acabam por esbarrar em dificuldades estruturais, como o desenho da matriz curricular que dispõe os diferentes conteúdos em disciplinas fragmentadas, com pouca integração vertical e horizontal. Os componentes 
básico e clínico são claramente identificados no organograma, além do distanciamento entre as disciplinas "de bancada" e a clínica e a cirurgia. ${ }^{6}$ Outro ponto importante das novas normativas diz respeito à organização do internato, estágio curricular obrigatório de treinamento em serviço com duração de dois anos, contemplando as grandes áreas de conhecimento (saúde coletiva, pediatria, clínica médica, cirurgia geral, saúde da mulher e saúde mental - recentemente incorporada como grande área), com atividades desenvolvidas nos diferentes níveis de atenção à saúde, foco na atenção primária e treinamento em urgência e emergência. O novo currículo preconizado deve ainda ser centrado no aluno, utilizando metodologias ativas de ensino e ferramentas de avaliação formativa, com atualização de projetos pedagógicos que contemplem as necessidades da comunidade., ${ }^{14} \mathrm{O}$ uso de ambiente virtual de aprendizagem bem como cenários de simulação (com uso de manequins ou pacientes padronizados) também está recomendado. Mais uma vez, as escolas tradicionais apresentam dificuldades para incorporar estas mudanças, mostrando certa inércia nas práticas pedagógicas. ${ }^{6,7}$

As recomendações estão dispostas e amplamente divulgadas, cabendo aos gestores organizar um ambiente que privilegie a reflexão sobre que caminhos escolher para alcançar os objetivos pactuados. Uma possibilidade que vem sendo apontada na literatura como catalizador de mudanças diz respeito ao desenvolvimento docente..$^{8,9} \mathrm{O}$ processo de aprimoramento curricular pode ser exitoso a partir da organização de atividades que mobilizem seus docentes, atores sem os quais não é possível legitimar nem implementar as transformações.

\section{Formação médica e a prática docente}

É possível traçar um paralelo entre as mudanças ocorridas no âmbito da educação médica e as atividades relacionadas à docência no âmbito da graduação. Como já comentado, no início do século XX o ensino era baseado principalmente no relatório Flexner que estabelecia padrões rigorosos de educação, preconizando o ensino em salas de aula, laboratórios, com ciclo básico destacado do clínico. ${ }^{1,10}$ Em meados do século foram percebidas expressivas inovações educacionais, com expansão do ensino superior para outros cenários para além dos hospitais escola e que possibilitavam o treinamento dos profissionais em formação. Nessa época surgiu também a ideia da pós-graduação médica por meio dos programas de residência e a integração com as áreas de pesquisa e assistência. Estas mudanças foram graduais, acompanhando o debate intenso na área, com ênfase nos aspectos relacionados à promoção da saúde. $\mathrm{O}$ enfoque atual seria no cuidado voltado para as necessidades do sujeito e da população, um currículo fundamentado em competências, com educação interprofissional alicerçada no trabalho em equipe, no domínio das tecnologias de informação e em habilidades de liderança e de gestão., ${ }^{410}$

Para se tornar professor, o jovem médico de tempos mais remotos se colocava na posição de aprendiz e desenvolvia suas habilidades de ensino repetindo as práticas utilizadas por seus bons professores e aquelas que resultaram positivas em sua própria experiência de aprendizado. A apreensão do conteúdo teórico (e prático) era suficiente para a docência e não havia uma preparação formal para esta atividade. ${ }^{11}$ Mesmo a expansão dos cursos de pós-graduação não resultou em capacitação docente para o ensino na graduação, pois acabou por favorecer a migração para atividades de pesquisa e a valorização de publicações científicas como indicador de mérito acadêmico. ${ }^{12}$ A máxima "publicar ou perecer" ganhou destaque cada vez maior e o campo da pesquisa foi reconhecido como atraente a partir da existência de dispositivos claros para ascensão e incentivos à progressão na careira.

A atuação de docentes não atualizados e sensibilizados aos novos rumos do ensino médico acaba colaborando para a manutenção da fragmentação de conhecimentos e a pouca participação discente no aprendizado. ${ }^{12,13}$ Os modelos utilizados estão baseados principalmente em aulas expositivas e avaliações somativas através de provas conteudistas que não abarcam outros aspectos para além da aquisição de conhecimentos teóricos, como o interesse do aluno, sua integração com a equipe e abordagem do paciente, entre outros. ${ }^{14}$ Os alunos se beneficiam de forma pontual com a experiência docente em sua área 
específica de atuação, com a possibilidade de interagir "na beira do leito" e discutir com profundidade apenas os casos clínicos acompanhados e disponíveis nas enfermarias ou atendidos nos ambulatórios. Novamente, não se garante com esta dinâmica uma visão integrada da situação de saúde e nem mesmo a necessária integração entre os diferentes conteúdos abordados ao longo da formação médica. ${ }^{8,14}$

Até hoje ainda vigora a preparação para a docência através da repetição de modelos e transmissão passiva de conhecimento, que não atende à multiplicidade de tarefas atuais. O professor não pode mais se limitar às aulas expositivas tradicionais ou palestras e precisa realizar atividades tão diversas quanto o já citado ensino na beira do leito e o uso de programas de computação para organização de testes e fóruns virtuais. Ao repertório relacionado ao ensino da graduação (ou em qualquer nível) precisam ser incorporadas a capacidade para atuar na perspectiva de facilitador de aprendizado e o planejamento e a avaliação de currículos, bem como a criação de material instrucional. ${ }^{8,14,15} \mathrm{O}$ docente pode se envolver em atividades de tutoria, avaliações estruturadas de habilidades clínicas, organização de portfólios, ensino em cenários e com pacientes simulados, construção de comunidades de prática, entre outras tantas metodologias ativas de ensino que privilegiam o saber do aluno e da comunidade. . $^{13,15}$ Claro está que não basta mais ser um bom médico para ser um bom professor. É preciso estar capacitado e, principalmente, comprometido com o processo de educar.

\section{Programas de desenvolvimento docente - definições e relevância}

A postura do docente frente às suas atividades acadêmicas na graduação é algo instigante. Quando atua como médico, a busca por conhecimento é uma rotina, bem como o constante aperfeiçoamento na sua prática diária para lidar com o paciente. Quando se interessa pela pesquisa, também pode ser observada a procura constante por novas maneiras de entender e abordar temas relevantes. No ensino, a atitude passiva e de repetição de práticas acadêmicas por vezes ultrapassadas não parece estar sintonizada com este profissional dinâmico, observador e que quer crescer. Através de programas de desenvolvimento docente é possível compreender melhor o que pode ser motivador e atrativo na docência e oportunizar processos de sensibilização do docente quanto à necessidade de atualização. A definição destes programas engloba qualquer atividade direcionada ao aperfeiçoamento do conhecimento e de habilidades nas áreas consideradas essenciais para o ensino nos diversos cenários da prática médica. ${ }^{8,16}$

Para a adequada estruturação das atividades com docentes, é preciso levar em consideração tanto os fatores de estímulo às práticas do ensino como aqueles que as dificultam. Para os docentes, parece ser importante perceber que a gestão (ou a direção da unidade) possui metas claras a cumprir com prazos estabelecidos e que suas atividades serão reconhecidas, valorizadas, com possível retorno financeiro para seu empenho. As barreiras apontadas pelos docentes para engajamento em atividades de capacitação se referem principalmente ao apoio institucional insuficiente, poucos recursos e estrutura para as mudanças nas práticas de ensino, além da sobreposição de múltiplas tarefas assistenciais e a dificuldade de reservar tempo protegido para o aperfeiçoamento das práticas de ensino..$^{14,17}$ Não se trata de falta de interesse, mas sim de dificuldade em perceber estas atividades como importantes. Aos gestores caberia demostrar que a aquisição destas habilidades relacionadas à docência não se limita apenas ao desenvolvimento individual e pode trazer múltiplos benefícios institucionais, facilitando inclusive o dia a dia da assistência desenvolvida no hospital, em ambulatórios, em salas de aula ou outros cenários.

A literatura aponta que nas últimas décadas o conceito de desenvolvimento docente foi se ampliando e passou a englobar, além das habilidades específicas do ensino, a melhor compreensão de currículos, a organização de feedback para os alunos, a utilização de ferramentas de avaliação formativas (por pares, pelos estudantes, autoavaliação, pelos gestores e pela comunidade) e aspectos relacionados à própria instituição. ${ }^{8,13}$ A ideia de profissionalismo tem sido incorporada nestes programas, com ênfase nas competências de liderança, uso de tecnologias avançadas e disponibilidade virtual da informação, diversidade social e 
melhores evidências para a educação médica. Os participantes se aproximam a partir da identificação de uma meta compartilhada e interesses comuns e trabalham em sintonia para alcançar os objetivos individuais e do grupo..$^{8,9,18}$

Algumas instituições já progrediram para a prática compulsória de engajamento nestes programas, relacionando a participação nas atividades ao mérito acadêmico e ascensão profissional. ${ }^{16,19}$ Para que estes avanços possam ocorrer, é necessário avaliar de forma cuidadosa quais as demandas dos docentes - o que e como fazer para atendê-los. As necessidades do sistema de saúde precisam estar contempladas, assim como o treinamento em habilidades de gestão e a ideia de pertencimento e aprimoramento institucional.

\section{Aprimoramento curricular na FCM - aproximando o docente da graduação}

Ao identificar os atores que vão fazer parte da revisão do novo e necessário currículo na FCM, a figura do docente acaba recebendo um papel de destaque. Esse profissional não só conhece profundamente as características de sua escola, onde muitas vezes percorreu parte de sua formação na graduação e durante a pós-graduação, mas também acaba participando da perpetuação dos modelos de aprendizagem que vêm sendo tradicionalmente vivenciados na instituição, sem a crítica necessária em relação à sua pertinência e atualidade.

A organização de um programa de desenvolvimento docente pode ser uma ferramenta eficiente para incrementar a vitalidade acadêmica, atendendo tanto a suas necessidades individuais como estruturando políticas institucionais que promovam a excelência no ensino da graduação. A opção da Direção da FCM por privilegiar estratégias para a sensibilização e o desenvolvimento do corpo docente como a principal ferramenta para alcançar as mudanças necessárias para o aprimoramento curricular tem alcançado boa receptividade. A Coordenação de Graduação vem desenvolvendo múltiplas frentes de trabalho, com a organização de seminários abordando temas de interesse na educação médica, a saber: ferramentas internas e externas de avaliação, mérito acadêmico, avaliação formativa no internato e integração entre ensino, serviço e comunidade. Os assuntos foram discutidos com a presença de palestrantes externos convidados, espaço para reflexão em plenária, seguido de momentos de confraternização. Também foram criados Grupos de Trabalho (GT), compostos por professores para revisão do projeto pedagógico, com a perspectiva da integração básico-clínico, da integração com serviços de saúde com a comunidade, do novo internato, da atenção ao aluno, de metodologias ativas e de ferramentas de avaliação formativas. A plataforma Moodle foi utilizada como ferramenta facilitadora de comunicação e como repositório de material teórico para o trabalho dos GT. ${ }^{20} \mathrm{~A}$ participação dos alunos foi incentivada em todas as iniciativas. Oficinas para desenvolvimento docente com foco na avaliação também foram organizadas, privilegiando a utilização de processos formativos no internato. Também foi possível elaborar uma proposta para o perfil do egresso, construída em parceria e disponibilizada para consulta pública e validação, trazendo transparência e legitimidade às etapas de aprimoramento curricular em curso na FCM.

\section{Consideração finais}

Docentes já sensibilizados, experiências inovadoras em curso e o apoio da gestão são fatores positivos que vêm facilitando todo o processo. $\mathrm{O}$ caminho é desafiante, exigindo parcerias institucionais e legitimação pelos pares. A ausência de dispositivos claros para a meritocracia acadêmica no âmbito da graduação parece ser uma lacuna na mobilização destes profissionais, fundamental para o alcance das metas relacionadas ao novo desenho curricular. A resposta às iniciativas tem sido encorajadora no âmbito da FCM, com a percepção de um movimento crescente de integração institucional e valorização das práticas na graduação. Através de atividades organizadas em parcerias, que possam aproximar e estimular os docentes e toda a comunidade acadêmica, pode ser possível melhorar o clima institucional, promovendo a construção de um currículo contemporâneo, centrado no aluno e articulado com as necessidades de saúde. A FCM segue sua trajetória histórica, garantindo o seu papel de destaque na formação de médicos diferenciados e contribuindo para a qualidade do sistema de saúde atual. 


\section{Referências}

1. Pagliosa F L, Da Ros M A. O Relatório Flexner: para o Bem e para o Mal. Rev Bras Educ Med. 2008;32(4):492-499.

2. Ministério da Educação. Parecer CNE/CES 1.133, de 7 de agosto de 2001. Diretrizes Curriculares Nacionais do Curso de Graduação em Medicina.

3. Instituto Nacional de Estudos e Pesquisas Educacionais Anísio Teixeira. Disponível em http://portal.inep. gov.br/enade. Acesso em: 04 nov 2014.

4. Ministério da Educação. Parecer CNE/CES 116, de 3 de abril de 2014. Diretrizes Curriculares Nacionais do Curso de Graduação em Medicina.

5. Mann K, Gordon J, Macleod A. Reflection and reflective practice in health professions education: a systematic review. Adv in Health Sci Educ. 2009; Oct 14(4):595-621.

6. Aguilar-da-Silva R H, Perim G L, Abdalla I G, Costa N M S C, Lampert J B, Stella R C R. Abordagens Pedagógicas e Tendências de Mudanças nas Escolas Médicas. Rev Bras Educ Med. 2009;33 (Supl 1):53-62.

7. Gianna Lepre Perim G L, Abdalla I G, Aguilar-da-Silva R H, Lampert J B, Stella R C R, Costa N M S C. Desenvolvimento Docente e a Formação de Médicos. Rev Bras Educ Med. 2009;33(Supl 1):70-82.

8. McLean M, Cilliers F, M van Wyk J. Faculty development: yesterday, today and tomorrow. AMEE Guide, Educ Manag. 2010; Series 2, 33.

9. Steinert Y, Naismith L, Mann K. Faculty development initiatives designed to promote leadership in medical education. A BEME systematic review: Guide no. 19. Med Teach. 2012;34:483-503.

10. Frenk J, Chen L, Bhutta Z A, Cohen J, Crisp N, Evans $\mathrm{T}$, et al. Health professionals for a new century: transforming education to strengthen health systems in an interdependent world. Lancet. 2010;376:1923-58.

11. Wilkerson L, Irby D M. Strategies for improving teaching practices: a comprehensive approach to faculty development. Acad Med. 1998;73(4):387-396.

12. Campos H H, Campos J J B, Faria M J S, Barbosa P F A, Araújo M N T. Programas de desenvolvimento docente em escolas médicas: oportunidades e perspec- tivas - mais do que uma necessidade. Cad ABEM. 2007; 3:34-38.

13. Mennin S, Kalishman S, Eklund M A, Friedman S, Morahan P S, Burdick W. Project-based faculty development by international health professions educators: practical strategies. Med Teac. 2013;35(2):e971-7. http://dx.doi.org/10.3109/014215 9X.2012.731096.

14. Harden R M, Crosby J. The good teacher is more than a lecture - the twelve roles of the teacher. Med Teach. 2000;22(4):334-347.

15. Foster H, Laurent R. How we make good doctors into good teachers: a short course to support busy clinicians to improve their teaching skills. Med Teach. 2013;35:4-7.

16. Steinert Y. Staff development for clinical teachers. ClinTeach. 2005;2(2):104-110.

17. Steinert Y, Mcleod P J, Bollat M, Meterissian A, Elizov M \& Macdonald M E. Faculty Development: a 'field of dreams'?. Medl Educ. 2009;43:42-49.

18. Cambridge D, Kaplan S, Suter V. Community of Practice Design Guide: A Step-by-Step Guide for Designing \& Cultivating Communities of Practice in Higher Education. Available from: http://net.educause.edu/ $\mathrm{ir} / \mathrm{library} / \mathrm{pdf} / \mathrm{nli0531.pdf}$. Access on: 11/4/2014.

19. Perlman R L, Christner J, Ross P T, Lypson ML. A successful faculty development program for implementing a sociocultural ePortfolio assessment tool. Acad Med. 2014; Feb, 89(2):257-62. http://dx.doi. org/10.1097/ACM.0000000000000120.

20. Plataforma Moodle. Avaiable from: http://moodle.org. br. Access on: 11/4/2014.

\section{Anna Tereza M. S. de Moura}

Departamento de Pediatria. Faculdade de Ciências Médicas. Universidade do Estado do Rio de Janeiro. Rio de Janeiro, RJ, Brasil.

\section{Albanita V. de Oliveira}

Departamento de Patologia e Laboratórios. Faculdade de Ciências Médicas. Universidade do Estado do Rio de Janeiro. Rio de Janeiro, RJ, Brasil. 